\title{
Uncertainty assessment of a dike with an anchored sheet pile wall using FEM
}

\author{
Aikaterini Rippi $^{1, a}$, Jonathan Nuttall ${ }^{2}$, Ana Teixeira ${ }^{1}$ and Timo Schweckendiek ${ }^{1,3}$ \\ ${ }^{1}$ Deltares, Unit Geo-engineering, Boussinesqweg 1, Netherlands \\ ${ }^{2}$ Deltares, Unit Software Centre, Boussinesqweg 1, Netherlands \\ ${ }^{3}$ Delft University of Technology, Mekelweg 2, Netherlands
}

\begin{abstract}
The Dutch design codes for the dikes with retaining walls rely on Finite Element Analysis (FEM) in combination with partial safety factors. However, this can lead to conservative designs. For this reason, in this study, a reliability analysis is carried out with FEM calculations aiming to demonstrate the feasibility of reliability analysis for a dike with an anchored sheet pile wall modelled in the 2D FEM, Plaxis. Sensitivity and reliability analyses were carried out and enabled by coupling the uncertainty package, OpenTURNS and Plaxis. The most relevant (ultimate) limit states concern the anchor, the sheet pile wall, the soil body failure (global instability) and finally the system. The case was used to investigate the applicability of the First Order Reliability Method (FORM) and Directional Sampling (DS) to analysing these limit states. The final goal is to estimate the probability of failure and identify the most important soil properties that affect the behaviour of each component and the system as a whole. The results of this research can be used to assess and optimize the current design procedure for dikes with retaining walls.
\end{abstract}

\section{Introduction}

Flood defence systems are large engineered systems protecting flood-prone areas from inundation. Since the consequences of failure of flood defences can be significant, it is worthwhile to analyse the reliability of such systems. In the Netherlands, after the safety assessment of 2011, it has been declared that $33 \%$ of the primary flood defences does not comply with the safety standards. As countermeasures, as far as the reinforcement of the dikes is concerned, several structural techniques have been introduced amongst which the most promising one is the construction of retaining walls inside the dikes. Such a technique can both improve the macro stability of the dike's inner slope and save space towards the inland part that needs to be habited. A special implementation of such a retaining wall is the anchored sheet pile wall, depicted in Fig. 1, that is often being applied in the Netherlands and it is going to be examined in this paper as a case study.

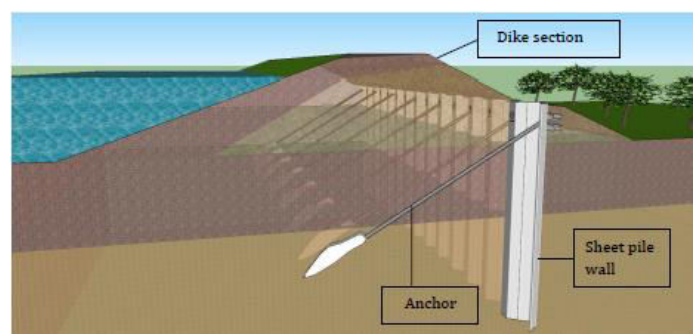

Figure 1. Schematized dike section with an anchored sheet pile wall
The Dutch design codes for the dikes with retaining walls modelled in FEM rely on the determination of partial safety factors [1]. Specifically, the load and resistance factor design (LRFD) approach is used. The LFRD code employs partial factors (e.g., resistance factors and load factors), which have been calibrated to achieve a target reliability index. In general, when applying a LRFD code for the design of a geotechnical structure, the nominal values of uncertain input parameters (e.g., resistance term and load term) should be selected (e.g., [2], [3]). However, the uncertainties in soil parameters and the applied loads, due to spatial variability and/or limited data availability, often make it difficult to quantify these values. Consequently, the predicted performance of the designed structure is also uncertain, that makes a conservative design to be more attractive. However, an overly conservative design tends to be cost-inefficient. Moreover, the recent Delta programme [4] includes new safety standards that are based on the evaluation of the acceptable flood risk (probability of failure (x) consequences) of each dike ring rather than only its strength towards a design load. This implies the need for incorporating more advanced probabilistic methods for design purposes.

Therefore, the incapability of the current design scheme to deliver an economic structure that satisfies the safety standards and simultaneously, the new requirements of the Delta programme makes it urgent that a new concrete design methodology is developed. For that purpose, in this research, the objective was to

\footnotetext{
$\overline{{ }^{a} \text { Corresponding author: katerina.rippi@deltares.nl }}$
} 
implement a full probabilistic analysis for evaluating the reliability of a dike reinforced with an anchored sheet pile wall modelled in FEM, so as to take into account all the possible uncertainties of the soil parameters and thus the uncertainties of the structure's performance.

This reliability analysis is an important step for incorporating the probabilistic methods in the design procedure by modelling the system performance in a reliable FEM software that diminishes the performance uncertainties. The implementation of such an analysis in this system can help to realize what the relevant, triggering failure mechanisms are and identify the most influencing parameters whose uncertainty plays a key role for the reliability of the structure. Additionally, the experience gained from this research can enhance the robustness and the efficiency of the future reliability methods.

In order to achieve that, a coupling between FEM and reliability methods has been applied. The most relevant (ultimate) limit states that were taken into account in this analysis, concern the anchor, the sheet pile wall and the soil body (global instability). In principle the probability of failure of each limit state and the most influencing parameters were estimated.

In previous studies, the influence of soil properties' uncertainties and the robustness of probabilistic methods have been also investigated in different type of structures (e.g., [5-8]). However, no one applied a reliability analysis on a complex system as a dike with a retaining wall is. The conclusions of the current research can contribute to design and optimization concepts and hopefully to a better understanding of the system behaviour.

The rest of this paper is organised as follows. Firstly, a brief description of the reliability analysis rationale and the reliability methods to be used in this paper is given. Secondly, the coupling procedure between the reliability methods and FEM is elaborated and explained. Thirdly, the case study whose reliability is to be evaluated, is presented and illustrated. Finally, the reliability analysis results are discussed and the estimated influencing soil properties are concluded for the specific case study. They are followed by concluding remarks and recommendations.

\section{Uncertainty assessment and reliability methods}

A first step of an uncertainty study can be described as "the definition of the problem". Initially, the variables of interest (or else the output variables) of which the uncertainty is to be quantified, shall be specified. In sequence, given several input variables for which the user may have data and/or expert/engineering judgment, a model denoted usually by a mathematical function should be introduced that enables the computation of the set variable of interest (system response).

After the general context has been staged, we should choose the criteria with which the uncertainty can be evaluated. The most complete measure of uncertainty, when dealing with a random vector, is the probability distribution. Therefore the statistical properties of the input variables shall be determined.

Eventually, the uncertainties of the input variables shall be translated in terms of uncertainty on the variables of interest. This procedure is called uncertainty propagation and can be carried out via several reliability methods (approximation methods or sampling methods). In the engineering world, this is also called reliability analysis. In particular, reliability describes the ability of a system or component to function under stated conditions for a specified time period.

In the consideration of the reliability of an element, the determination of the probability of failure is the central issue. The limit between failure and non-failure is defined as a limit state and the reliability is the probability that this limit state is not exceeded. The limit states are interpreted through the so-called limit state functions (LSF) whose general form is $Z=R-S$, where $R$ is the resistance term and $S$ is the load term.

In this research, the First Order Reliability method (FORM) and the Directional Sampling method (DS) are going to be implemented. The latter is considered to be a fully probabilistic method whereas the former a fully probabilistic method with approximations. The probabilistic tool that is utilized in this research and from which the aforementioned methods are available is OpenTURNS (OT), which is a scientific library with a python module dedicated to treatment of uncertainties.

Last but not least, a better understanding of uncertainties' influence can be achieved by analysing the contribution of the different uncertainty sources to the uncertainty of the variables of interest via a sensitivity analysis. In both FORM and DS methods, the importance factors (or else the $a$-values) are estimated as a quantitative measure of a local sensitivity analysis which indicate the most influencing random variables for the system's response.

\subsection{First Order Reliability method (FORM)}

As far as the FORM is concerned, the concept is based on the approximation of the LSF with a linear polynomial after the random variables being transformed into the normalized space [9]. The first step of this method is the transformation of the random variables to equivalent standard normally distributed variables and the whole procedure is carried out in $u$-space (or else standard space). For a set of random variables $X=\left(x_{1}, x_{2}, \ldots, x_{n}\right)$ that are normally distributed this step is as follows:

$$
u_{i}=\frac{x_{i}-\mu_{x_{i}}}{\sigma_{x_{i}}}, i=1,2, \ldots, n
$$

where $u_{i}$ is the transformed $x_{i}$ in the normalized space, $\mu_{x_{i}}$ is the mean value of the random variable and $\sigma_{x_{i}}$ is its standard deviation.

In the sequence, the limit state function, $Z$, is expressed in terms of $U=\left(u_{1}, u_{2}, \ldots, u_{n}\right)$ so as $Z=g(U)$. The second step is the approximation of the function with the first two terms of the Taylor-polynomial. The approximation reads: 


$$
\begin{gathered}
Z=g(\boldsymbol{U}) \Leftrightarrow \\
Z \approx g\left(U_{o}\right)+\sum_{i=1}^{n} \frac{\partial g}{\partial u_{i}}\left(U_{o}\right)\left(U_{i}-U_{o_{i}}\right)
\end{gathered}
$$

where $\vec{U}_{0}$ is the point where the linearization takes place. This approximation of $Z$ is linear and according to the central limit theorem it is normally distributed. The expected value of the LSF can be approximated by the expected value of the linearized function. The reliability index, $\beta$, is defined equal to the minimum distance from the origin to the design point [9]:

$$
\beta=\min _{z=0}(\|U\|)
$$

Looking for the design point is basically an optimization problem. The obtained $\beta$-value is used to determine a new point, in which the LSF is linearized. In this case the importance factors, $\alpha_{i}$, are calculated as:

$$
a_{i}=\frac{\frac{\partial g}{\partial x_{i}}\left(U^{-}\right) \sigma_{U_{i}}}{\sqrt{\sum_{j=1}^{n}\left(\frac{\partial g}{\partial x_{j}}\left(V^{-}\right) \sigma_{U_{j}}\right)^{2}}}, \quad \sum_{i=1}^{n} a_{i}^{2}=1
$$

where $U^{*}$ is the design point and $\mathrm{j}=1, \ldots, \mathrm{n}$ is the number of variables. $a_{i}$ expresses the contribution of the variance of each variable to the total variance of $Z$, in the design point. In Fig. 2, an illustration of the method is depicted.

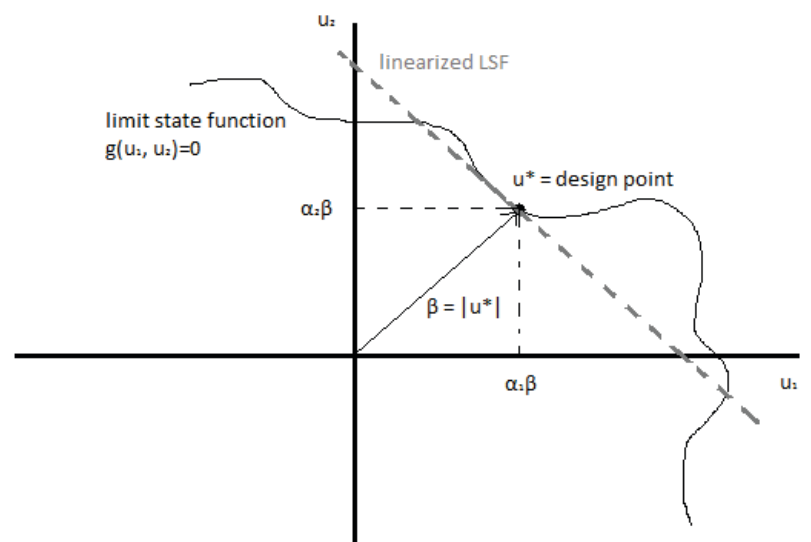

Figure 2 Two-dimensional illustration of $\mathrm{u}$-space, LSF and design point

The probability that $\mathrm{Z}<0$ (i.e. probability of failure, $\left.P_{f}\right)$ can be determined using the standard normal cumulative density function:

$$
P_{f, F O R M}=P(Z<0)=\Phi\left(-\frac{\mu_{z}}{\sigma_{z}}\right)=\Phi(-\beta)
$$

\subsection{Directional Sampling method (DS)}

Regarding DS, it is a method in which "directions" are sampled. For each sampled direction, it is evaluated whereas along the line of a certain direction, the LSF ( $Z$ ) equals to zero or not. This procedure is repeated for a number of directions and this method is applied in the standard normal space ( $u$-space). The maximum length of the direction line as well as the step size along the direction can be steered in order to increase the efficiency of the method and presumably decrease the computational time. The probability of failure can be calculated as follows:

$$
P_{f, D s}=\frac{1}{N} \sum_{i=1}^{N} q_{i}
$$

where $N$ is the number of sampled directions and $q_{i}$ is the probability that $Z \leq 0$ along the $i^{\text {th }}$ direction, estimated according to the chi-squared distribution [17].

A method was developed in order to estimate the $\alpha^{2}$ values of the random variables out of a DS analysis as OT does not have an available method so far. After the probabilistic analysis with OT, the output samples are only available in the $x$-space. For that purpose, a transformation of the output samples to $u$-space was firstly carried out. Then, the distance to the origin of all samples (in $u$-space) that are located on a direction where failure $(Z=0)$ was detected, is calculated as follows:

$$
\left|u_{i}\right|=\sqrt{\sum_{j=1}^{n} u_{i j}^{2},} \quad i=1 \ldots
$$

where $n$ is the number of random variables and $u_{i j}$ is the $i^{t h}$ "failure" sample of the $j^{t h}$ random variable. $\left|\mathbf{u}_{\mathrm{i}}\right|$ is equivalent with the reliability index of this direction, $\beta_{i}$. The $\alpha$-values for each random variable can then be calculated as:

$$
\alpha_{j}=-\frac{u_{j}}{\beta i}
$$

After that, three different methods were studied [14] in order to evaluate the influence of the random variables on the response of the LSF, i.e. the $\alpha^{2}$-values (square of Eq. 8):

- Shortest distance, " $\beta_{\min } "$

- Average $10 \%$

- Average all

In the Shortest distance method, the sample with the smallest distance to the origin is considered to be the design point and thus the corresponding influence factors are estimated according to that point. The smallest distance is also equivalent to the reliability index, $\beta_{\text {min }}$. This method is well-known for estimating the design point out of MC techniques [10]. However, for a soil failure modelled in FEM, this might not be always indicative of the real influence that the soil properties can have on the system failure. Therefore, the last two techniques were used as additional in order to get a better insight into the dominant soil properties.

The second method, Average 10\%, takes into account the $\alpha^{2}$-values of the samples in the failure domain whose distance to the origin lies within the $10 \%$ higher than $\beta_{\text {min }}$ and averages them. Therefore, it is firstly required the calculation of $\beta_{\min }$ as it was described for the Shortest distance method. Such a technique helps to identify the 
other directions that are close to the one which gives the shortest distance to the origin and thus inspect if the important variables that were indicated according to the first method are influential for other failure mechanisms as well.

Last but not least, the third method, Average all, averages the $\alpha^{2}$-values of all the samples located in the failure domain. This shows the overall contribution of the random variables to the response of the LSF under investigation where multiple failure mechanisms are involved.

In Fig. 3, the aforementioned methodology is summarized.

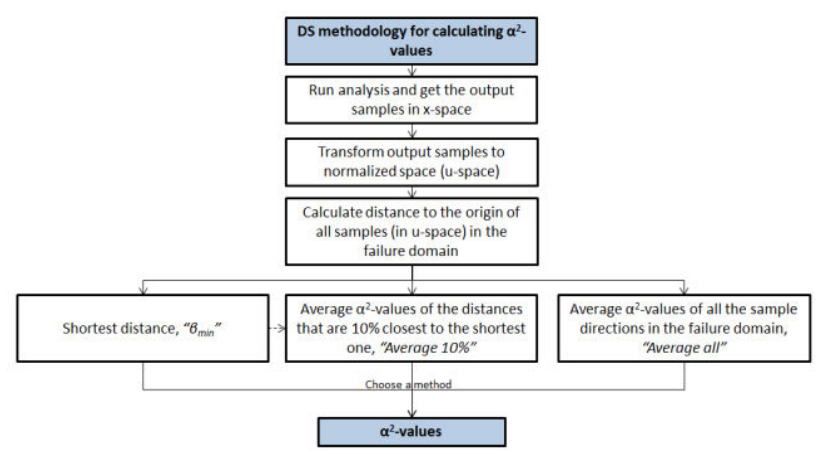

Figure 3 DS methodology for calculating $\alpha 2$-values

\section{Coupling Reliability methods with FEM}

The finite element simulation will be carried out in Plaxis 2015. Plaxis is a finite element software for plane strain and axi-symmetric modelling of soil and rock behaviour. Moreover, it supports a fully automatic mesh generation, allowing for a virtually infinite number of 6node and 15-node elements.

The coupling of reliability analysis and FEM requires an interface for the communication between each other. When a reliability tool is coupled with another software program, the reliability program carries out the whole reliability analysis and it uses the other program only for the evaluation of the limit state function. More precisely, OT interface should be able to amend the input random variables and read Plaxis output values for important variables such as material parameters, pore pressures generation and stresses development and corresponding deformations inside the dike. Respectively, Plaxis has to be also capable of obtaining the new values that has been set by OT for the variables that are treated as stochastic during an iterative process according to the reliability assessment. In Fig. 4, an illustration of the coupling methodology and its function is shown.

In principle, an input file is firstly required where the user set the preferable reliability method to be used, the stochastic input parameters and their probability distributions, the joint probability distribution and the corresponding correlation matrix and finally the limit state function is formed depending on the situation. However, such input files should be interpreted so as to be readable by both Plaxis and OT. Therefore, an input interpreter was created which is actually a python script that helps OT to start up the reliability analysis according to the assigned method, variables, distributions and LSF. As it was mentioned before, the evaluation of the limit state function is conducted by Plaxis. For that purpose, the input interpreter should be also able to send the next set of input parameters to Plaxis. However, an additional means of connecting the interpreter with Plaxis is also needed. This can be achieved via a Plaxis interface which actually calls Plaxis to make the calculations while it also transfers the required value of the limit state function to input interpreter and this in turn to OT.

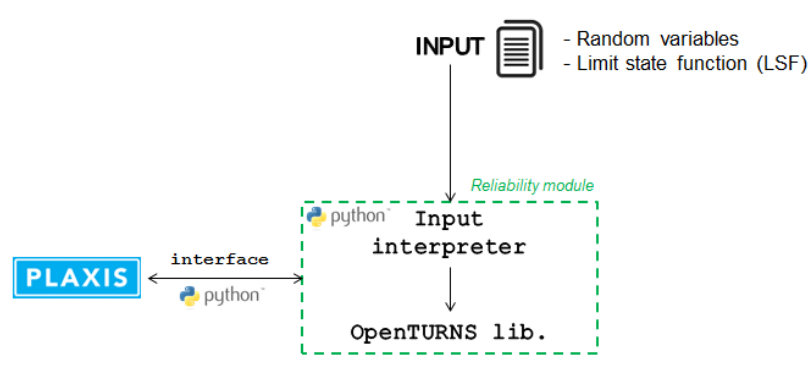

Figure 4 Coupling OpenTURNS-Plaxis

\section{Case Study and random variables}

In Fig. 5, the case study as it has been modelled in Plaxis $2 \mathrm{D}$ is depicted. The sheet pile has been modelled as an elastic plate element whereas the anchor as an elastic fixed-end-anchor element. For all the steel members, a Young's modulus of E $=210 \mathrm{GPa}$ was used. As far as the soil structure is concerned, i.e. the dike section, it consists of two soil materials which are mainly sandy clay whereas the foundation layers can be identified as a top clay layer and a bottom sand layer. The constitutive model that was chosen in this research was MohrCoulomb. This model can successfully identify the potential failure modes and simulate the soil behaviour until failure. However, for the analysis of soft soils deformations and settlements around a wall, more advanced constitutive models are recommended (i.e. Hardening Soil model, Soft Soil model etc). In that research, a more advanced constitutive model, such the Hardening Soil model, was not used due to its complexity and the results' randomness in Plaxis calculations.

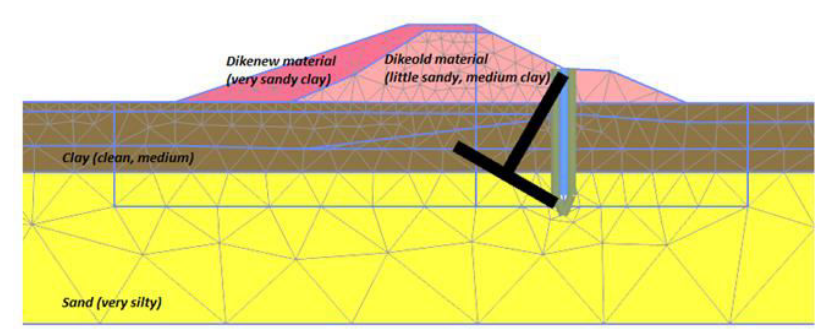

Figure 5 Case study: Plaxis mesh and soil layers

The properties of the soil layers are shown in Table 1. More precisely, in Table 1, the corresponding probability distribution ( $\mathrm{TN}=$ Truncated Normal, LN=Lognormal, $\mathrm{N}=$ Normal) together with the mean values $\left(1^{\text {st }}\right.$ number in the brackets) for the soil properties of each layer are given and the coefficient of variation $\left(2^{\text {nd }}\right.$ number in the 


\begin{tabular}{ccccc}
\hline & Clay & Sand & Dikeold & Dikenew \\
\hline$\varphi\left[^{\circ}\right]$ & $\mathrm{TN}(21,10 \%)$ & $\mathrm{TN}(36,10 \%)$ & $\mathrm{TN}(33.5,10 \%)$ & $\mathrm{TN}(34.7,10 \%)$ \\
$\mathrm{c}[\mathrm{kPa}]$ & $\mathrm{LN}(14,20 \%$ & $\mathrm{LN}(0,20 \%$ & $\mathrm{LN}(3.67,20 \%$ & $\mathrm{LN}(5.64,20 \%$ \\
$\mathrm{G}[\mathrm{kPa}]$ & $\mathrm{LN}(1190.2,25 \%)$ & $\mathrm{LN}(12300,25 \%)$ & $\mathrm{LN}(1740.67,25 \%)$ & $\mathrm{LN}(967,25 \%)$ \\
$\mathrm{R}_{\text {inter }}[-]$ & $\mathrm{TN}(0.5,20 \%)$ & $\mathrm{TN}(0.66,20 \%)$ & $\mathrm{TN}(0.5,20 \%)$ & $\mathrm{TN}(0.5,20 \%)$ \\
$\gamma_{\text {unsat }}\left[\mathrm{kN} / \mathrm{m}^{3}\right]$ & $\mathrm{N}(18.5,5 \%)$ & $\mathrm{N}(21,5 \%)$ & $\mathrm{N}(20.7,5 \%)$ & $\mathrm{N}(18.5,5 \%)$ \\
$\mathrm{v}[-]$ & $\mathrm{LN}(0.35,10 \%)$ & $\mathrm{LN}(0.30,10 \%)$ & $\mathrm{LN}(0.35,10 \%)$ & $\mathrm{LN}(0.35,10 \%)$ \\
\hline
\end{tabular}

Table 1 Statistical characteristics of the soil parameters

brackets) that were considered. In particular, the soil properties that were considered in this paper is the friction angle $(\varphi)$, the cohesion $(c)$, the shear stiffness $(G)$, the interface strength between the soil and the structure $\left(R_{\text {inter }}\right)$, the soil unsaturated unit weight $\left(\gamma_{\text {unsat }}\right)$ and the Poisson ratio $(v)$. The statistics were chosen arbitrarily but in a realistic range according to the Dutch code for geotechnical structures. The distribution types and the coefficients of variation for each parameter were chosen according to the knowledge that has been obtained until now about the physically possible ranges of such parameters and the recommendations that have been given in several researches [5, 8, 11, 12, 13]. Particularly for the saturated and unsaturated volumetric weight, $\gamma_{\text {sat }}$ (saturated unit weight) and $\gamma_{\text {unsat }}$ respectively, a relationship was established in order to derive the one from the other. In general, $\gamma_{\text {unsat }}$ varies between the real dry weight and $\gamma_{\text {sat }}$ depending each time on the degree of saturation. An estimated maximum difference between $\gamma_{\text {unsat }}$ and $\gamma_{\text {sat }}$ is approximately $4 \mathrm{kN} / \mathrm{m}^{3}$. In this research, $\gamma_{\text {sat }}$ was expressed as the summation of $\gamma_{\text {unsat }}$ and a variable with uniform distribution in the range of $[0,2]$.

It should be mentioned that the soil properties were considered to be independent from each other. However, a further investigation is recommended with correlated soil variables, so as the correlation effect on the reliability of the system to be evaluated.

\section{Failure mechanisms and limit state functions}

For a reinforced dike with retaining walls, there are basically three classes of structural elements:

- Retaining wall (i.e. sheet piles);

-Anchors;

- Soil structure (i.e. dike section).

For the reliability analysis of each of the elements, the probability of failure was determined. In the design process, one is most interested in the Ultimate Limit State (ULS) of a failure mechanism. This state describes the situation wherein the acting extreme loads are just balanced by the strength of the construction. If that limit state is exceeded the construction will lose its functionality and thus collapse or fail.

The most relevant failure mode for the sheet pile wall and the anchor is the exceedance of the yield strength which corresponds to the ultimate steel strength. As far as the sheet pile wall is concerned, the response of the structure is mainly due to bending moment and the axial forces (where an axial force is present, allowance should be made for its effect on the moment resistance). Therefore, the LSF can be formed as the difference between the maximum developed stress and the yield stress, $\sigma_{y}[\mathrm{kPa}]$ (see Eq. 10).

As far as the soil failure is concerned, in this research, emphasis is given on the dike global instability which actually consists of several failure modes, the combination of which can lead to the overall instability of the dike and thus to soil body failure. The main failure mechanisms are:

- Overflow/overtopping

- Inner/outer slope macro instability

- Horizontal sliding

- Micro instability

- $\quad$ Piping

However, Plaxis is not capable of accounting for all of them. More precisely, Plaxis assumes the soil to be a continuous body and thus it can model movements at the scale of soil bodies, but not on a soil particle level. Moreover, Plaxis cannot deal with the flow and waves occurring in "open water", i.e. water outside the soil in a canal, a lake or sea for instance. Therefore, piping, micro instability and overflow/overtopping have not been considered.

For the rest of the failure modes, soil failure is defined as shear failure and can take several patterns depending on the location and the source of the trigger mechanism [14]. In this paper, the total probability of failure of the soil body due to global instability is to be determined. This is due to the fact that the application of FEM is capable of simulating the combination of these failure patterns and thus the overall failure can be evaluated.

The LSF for the soil failure can be formulated in different ways [5]. After considering the possibilities and limitations of each formulation [14], the Plaxis definition of soil collapse was used in order to assess the failure. In that case, if soil failure is detected from Plaxis, an error message of "Soil collapses" appears. This happens when the total specified load has not been applied. In physical terms this means that the current value of the stiffness parameter (CSP) is less than 0.015 [15]. CSP is a measure for the amount of plasticity that occurs during the calculation. When the solution is fully elastic, CSP is equal to unity whereas at failure it approaches zero. Therefore, soil reaches its upper limit of plasticity and it collapses which can be visualized as a settlement of the dike body. Such a warning in the analysis is thus assumed to be a possible failure situation. The LSF can be then written as follows: 


$$
Z=\left\{\begin{aligned}
-1, & \text { if computation is unsuccessful } \\
1, & \text { if computation is success ful }
\end{aligned}\right.
$$

Considering the above, the system failure, in this research, is considered as a serial system of the anchor, sheet pile wall and soil body failures. In a serial system, failure is considered when one of the components fails. Thus, the general LSF was formulated as the minimum of the three LSFs for the soil, the sheet pile and the anchor and it is given below:

$$
Z=\min \left\{\begin{array}{c}
\text { check if soil fails }[1 \text { or }-1], \\
\sigma_{y, s p}-\max \left[\left(\frac{M(z)}{W_{a l}}+\frac{F_{n}(z)}{A_{s p}}\right)\right] \\
\sigma_{y, a}-\frac{N_{a}}{A_{a}}
\end{array}\right.
$$

As it can be recognised in Eq. 10, the top part is identical to Eq. 9 for the soil failure whereas the last two are for the sheet pile and the anchor respectively. In the middle one, for the sheet pile, $M(z)$ and $F_{n}(z)$ is the bending moment and the axial force over the sheet pile depth, respectively. $W_{e l}\left[\mathrm{~m}^{3}\right]$ is the elastic section modulus and $A_{s p}\left[\mathrm{~m}^{2}\right]$ is the cross sectional area of the sheet pile wall. In case of the anchor, $N_{\alpha}[\mathrm{kN}]$ is the calculated anchor force and $A_{\alpha}\left[\mathrm{m}^{2}\right]$ is the cross sectional area of the anchor. Finally, $\sigma_{\mathrm{y}, \mathrm{sp}}$ and $\sigma_{\mathrm{y}, \mathrm{a}}$ is the yield stress of the sheet pile and the anchor respectively. In this paper, the results of a FORM analysis as they were deduced in case of the anchor failure as a separate mechanism are presented as well as the results of a DS analysis for the system as a whole.

It should be mentioned at this point, that the probability of failure of the system, computed in this paper, differs from the overall probability of flooding which takes into account any potential failure mechanism of the system combined with the uncertainties of the water elevation. More precisely, as far as the dike safety is concerned, a probability of failure, $P\left(Z_{i}<0 \mid h_{i}\right)$ (where $Z$ is the LSF), under a certain water level, $h_{i}$, is estimated which in sequence is multiplied with the occurrence probability of the corresponding water level, $f\left(h_{i}\right)$ (i.e. it is the PDF of $h_{i}$ ), in order to estimate the overall probability of failure. Then, that product is accumulated over a required range of water levels that can jeopardise the overall stability of the structure, in order to calculate the overall probability of failure, $P_{f t}$, or else the probability of flooding. This probability is then compared to the one established from the safety standards so as to reassure the safety or not of the structure. $P_{f t}$ can be estimated as follows [18]:

$P_{f t}=\int_{h_{i=1}}^{h_{n}} P\left(Z_{i}<0 \mid h_{i}\right) f\left(h_{i}\right) d h \approx \sum_{i} P\left(Z_{i}<0 \mid h_{i}\right) f\left(h_{i}\right) d h$

In this research, the probability $P\left(Z_{i}<0 \mid h_{i}\right)$ is to be estimated where $h_{i}$ is a specific water level and especially the design water level, as it is considered to be the most challenging part of the procedure described above. After setting up the steps and implementing them successfully for the estimation of $\left(Z_{i}<0 \| h_{i}\right)$, the calculation of the overall probability of failure is just a repetition of the same procedure for more water levels. It should be mentioned that in this paper, failure consists only of the macro-instability failure mode whereas the rest are excluded for the time being.

\section{Reliability analysis results}

Before starting analysing the reliability of the system, it is important to choose what will be the stochastic variables for the different LSFs. Due to the high amount of soil properties, a preliminary global sensitivity analysis was applied in order to filter out and if possible, to reduce the random variables so as the reliability analysis to be more efficient and computationally accessible [14]. The parameters that were eventually considered as stochastic in each LSF are summarized in Table 2 and they were chosen according to both the sensitivity analysis and the engineering judgment. The statistical properties of the

\begin{tabular}{|c|c|c|c|c|}
\hline & Clay & Sand & Dikeold & Dikenew \\
\hline$\gamma_{\text {unsat }}$ & S, SP & S & SP & S \\
\hline$c$ & S, A, SP & & $\mathrm{S}, \mathrm{A}, \mathrm{SP}$ & S \\
\hline$\varphi$ & $\mathrm{S}, \mathrm{A}, \mathrm{SP}$ & $\mathrm{S}, \mathrm{A}, \mathrm{SP}$ & $\mathrm{S}, \mathrm{A}, \mathrm{SP}$ & $\mathrm{S}$ \\
\hline$G$ & $\mathrm{~S}, \mathrm{~A}, \mathrm{SP}$ & $\mathrm{S}, \mathrm{A}, \mathrm{SP}$ & A, SP & \\
\hline$v$ & $\mathrm{~A}$ & $\mathrm{~A}$ & A, SP & \\
\hline$R_{\text {inter }}$ & A, SP & $\mathrm{A}$ & A, SP & \\
\hline
\end{tabular}
variables are shown in Table 1.

$\mathrm{LSF}(\mathrm{S}=$ soil failure, $\mathrm{SP}=$ sheet pile failure, $\mathrm{A}=$ anchor failure)

For all the three classes of structural elements and the system as a whole, an evaluation of their LSF was carried out by Rippi [14]. From this assessment, the probability of failure as well as the importance factors was deduced. Such an analysis contributed to acquiring a better understanding on the soil-structure interaction as well as the behaviour of the structural elements and the soil body separately. In the next subsections the results from the anchor failure and the soil failure are presented as a demonstration of FORM and DS performance respectively while in the end the results of the system reliability analysis are discussed. The probabilistic analysis of the sheet pile wall is presented in detail elsewhere [14].

\subsection{Anchor failure}

For the anchor failure, the LSF was formulated as follows:

$$
Z=\sigma_{y}-\frac{N_{a}}{A_{a}}
$$

where $\mathbb{N}_{\mathrm{a}}[\mathrm{kN}]$ is the anchor force, $\sigma_{\mathrm{y}}=450 \mathrm{kPa}$ is the yield stress and $A_{a}=933 \mathrm{~mm}^{2}$ the cross section area. These structural properties were initially defined by a deterministic analysis [14]. The reliability method to be utilized in this analysis is FORM and the random variables to be used, are listed in Table 2 with $A$ index. In 
Table 3, the results of the reliability analysis with FORM are listed. Additionally, the design point together with the importance factors of each variable is presented. It should be mentioned at that point that the sensitivity analysis helped to define a starting point closer to the design point and thus reduce the computational time required for the analysis. More precisely, the analysis lasted approximately 1.6 hours whereas by starting from mean values, from which by default FORM starts the iterations, it would take almost a day.

\begin{tabular}{lc}
\hline \hline FORM $\boldsymbol{P}_{\boldsymbol{f}}$ & $2.84 * 10^{-6}$ \\
$\beta$ & 4.5 \\
Number of LSF calls & 119 \\
Maximum number of & 100 \\
iterations & 1.6 \\
Elapsed time (hr) & \\
\hline \hline
\end{tabular}

Table 3 Reliability results for the anchor failure

The influence coefficients in Fig. 6 indicate that this limit state is governed by the shear stiffness of the clay layer, G. Furthermore, the shear stiffness of the Dikeold material seems to contribute significantly to the anchor yield stress exceedance. It can be concluded that the problem is still in the elastic domain as far as the soil behaviour is concerned. In case of predominately plastic behaviour, the strength properties of the soil ( $\varphi$ and $c)$ become more important. In Fig. 7, a demonstration of the design point as it was acquired by the FORM analysis is presented. The figures indicate that presumably due to the clay layer withdrawal in the passive side as it is depicted in Fig. 7 with the red shadings, sheet pile moves to the right and subsequently anchor is tensioned enough to reach its yield stress.

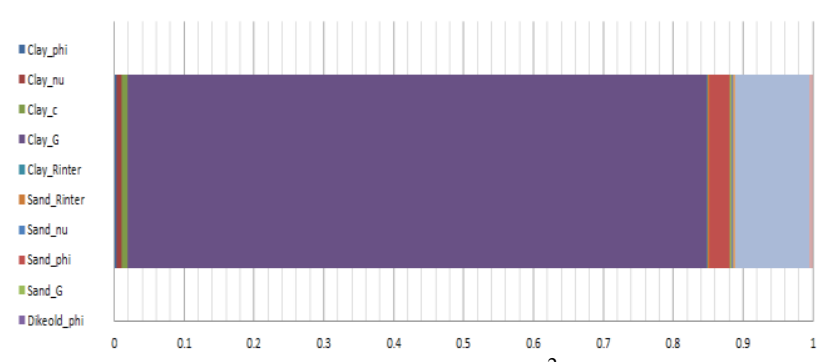

Figure 6 Estimated importance factors $\alpha^{2}$ for anchor failure with FORM

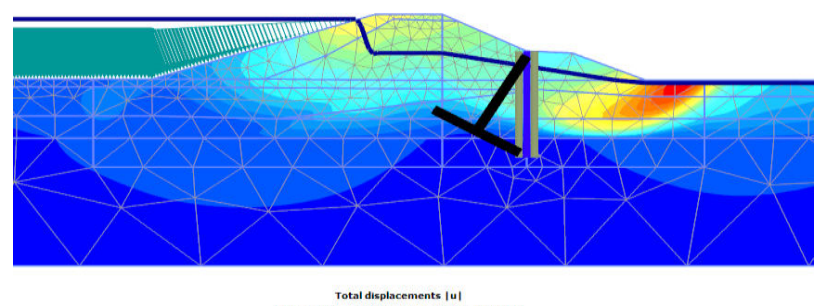

Figure 7 Total displacements due to the anchor failure (in red shadings the maximum total displacements are indicated). Potential developed failure mechanism due to the anchor weakening

The calculations were repeated with the 6 most influencing parameters both with FORM and DS in order to investigate the effect of reducing the number of random variables and in order to validate the results of FORM analysis. In Table 4, the reliability results of respectively FORM and DS are presented while in Table 5 the importance factors of both methods are juxtaposed (the darker the cell is, the higher the importance factor is).

\begin{tabular}{l|cc}
\hline \hline & FORM & DS \\
\hline \hline $\boldsymbol{P}_{\boldsymbol{f}}$ & $8.5 * 10^{-6}$ & $1.5 * 10^{-5}$ \\
$\beta$ & 4.3 & 4.2 \\
$\begin{array}{l}\text { Number of } \\
\text { LSF calls }\end{array}$ & 28 & 776 \\
$\begin{array}{l}\text { Maximum } \\
\text { number of } \\
\text { iterations }\end{array}$ & 100 & 100 \\
$\begin{array}{l}\text { Elapsed time } \\
\text { (hr) }\end{array}$ & $\begin{array}{l}\text { 0.34 (start close } \\
\text { to design point) }\end{array}$ & 11.5 \\
\hline \hline
\end{tabular}

Table 4 Reliability results of FORM and DS with the most important parameters for the anchor failure

\begin{tabular}{l|cc}
\hline \hline & FORM & DS \\
\hline \hline \multirow{2}{*}{$v_{\text {clay }}$} & 0.014 & 0.059 \\
$c_{\text {clay }}$ & 0.001 & 0.045 \\
$G_{\text {clay }}$ & 0.860 & 0.651 \\
$\varphi_{\text {sand }}$ & 0.012 & 0.042 \\
$G_{\text {dikeold }}$ & 0.109 & 0.134 \\
Rinter $_{\text {dikeold }}$ & 0.004 & 0.069 \\
\hline \hline
\end{tabular}

Table 5 Estimated importance factors: FORM comparison with DS

As it can be noticed, comparing FORM results from Table 3 and 4 , the reliability index changes from $\beta=4.5$ with 14 parameters to $\beta=4.3$ with the 6 most influencing parameters. This verifies the importance of the most influencing parameters that it was found in the analysis shown in Table 3. The small discrepancy though between the two reliability indexes might be attributed to the interaction effect of the soil variables on the output performance.

The estimation of failure probability with DS is almost the same with FORM which means that FORM estimations are reliable. As far as the importance factors are concerned, DS also came up with the conclusion that the shear stiffness of the clay and the Dikeold material are the most crucial soil properties for the anchor stress level. However, according to DS the stiffness of clay, $v$ and the interface strength, $R_{\text {inter }}$ between the Dikeold material and the sheet pile wall seem to also contribute to the limit state of the anchor.

The reason why the strength of the clay layer under the dike and the Dikeold material (the part of the dike that comes in contact with the anchor and the sheet pile wall) are the most important variables, can be explained by the principle of soil arching. According to this principle, the soil columns on both sides of the rigid sheet pile wall are more compressive than the soil columns on the top of the wall (i.e. overlying dike body) because of the higher stiffness of the wall when compared with soils. As such, soil columns on both sides tend to settle more than the soils on top of the rigid wall and this differential settlement causes a downward shear force acting along the sides of soil columns on top of the wall. As such, the 
vertical load on the wall becomes larger than the sole weight of soil columns on its top and the anchor that actually receives the most of this vertical load reaches eventually its yielding stress.

\subsection{Soil failure}

The LSF for the evaluation of the soil failure is given in Eq. 9 and the reliability method that was chosen in that case is DS due to the formulation of the limit state function and the convergence issues of FORM. More precisely, FORM needs an answer from the limit state function in order to proceed with the next iteration. Since the soil failure limit state function only returns $-1 / 1$ (failure/non-failure), FORM analysis cannot converge and thus DS method has been preferred.

The results of the reliability analysis according to DS are presented below. In particular, 300 iterations were carried out and 12 input stochastic soil parameters were used, indicated in Table 2 with the $S$ index. Specifically, in Table 6, the probability of failure, the reliability index, the number of iterations and the duration of the analysis are shown.

\begin{tabular}{lc}
\hline \hline DS $\boldsymbol{P}_{\boldsymbol{f}}$ & $1.3 * 10^{-8}$ \\
$\beta$ & 5.5 \\
Number of LSF calls & 1840 \\
Number of iterations (or & 300 \\
directions) & 34 \\
Elapsed time (hr) & 34 \\
\hline \hline
\end{tabular}

Table 6 Reliability analysis' results of DS for the soil failure

In Table 7, the importance factors for the several soil properties are presented as they were estimated from the different methods presented in section 2.2. As someone could observe, the results of each method shows the same tendency concerning the most influencing variables, with some discrepancies though. These deviations of the $\alpha^{2}$ values were investigated [14] and it was found that Plaxis failed calculations can be attributed to mainly numerical errors different from the "Soil Collapses". These errors shall be considered as unrealistic failure mechanisms and the corresponding samples should be excluded from the analysis. In Fig. 8, an illustration of a potential failure mechanism according to the deduced design point is shown.

\begin{tabular}{l|ccc}
\hline \hline & $\beta_{\min }$ & Average 10\% & Average all \\
\hline \hline$\varphi_{\text {dikenew }}$ & 0.03236 & 0.03236 & 0.03958 \\
$c_{\text {dikenew }}$ & 0.00002 & 0.00002 & 0.00631 \\
$\gamma_{\text {dikenew }}$ & 0.00431 & 0.00431 & 0.09052 \\
$\varphi_{\text {sand }}$ & 0.20591 & 0.20591 & 0.11071 \\
$G_{\text {sand }}$ & 0.07206 & 0.07206 & 0.05299 \\
$\gamma_{\text {sand }}$ & 0.02851 & 0.02851 & 0.02699 \\
$G_{\text {clay }}$ & 0.01356 & 0.01356 & 0.05572 \\
$c_{\text {clay }}$ & 0.01098 & 0.01098 & 0.10848 \\
$\varphi_{\text {clay }}$ & 0.01131 & 0.01131 & 0.17248 \\
$\gamma_{\text {clay }}$ & 0.43123 & 0.43123 & 0.17271 \\
$c_{\text {dikeold }}$ & 0.12658 & 0.12658 & 0.09913 \\
$\varphi_{\text {dikeold }}$ & 0.02755 & 0.02755 & 0.03114
\end{tabular}

Table 7 Estimated importance factors $\alpha^{2}$ for soil failure with the different methods

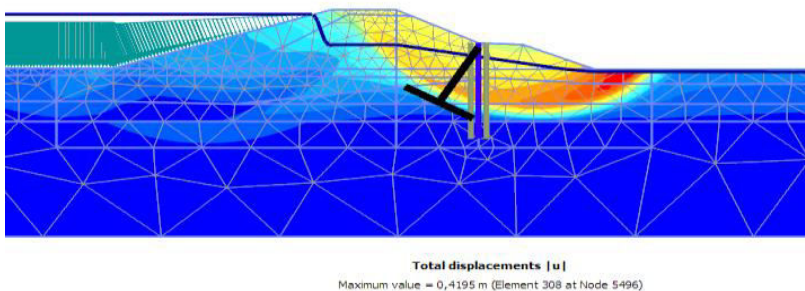

Figure 8 Total displacements due to the anchor failure (in red shadings the maximum total displacements are indicated).

Potential developed failure mechanism due to the soil collapse

From a general perspective, the soil properties of the clay layer seems to be determinant, whereas also sand and Dikeold material play an important role to the soil failure. More precisely, according to the Average all method, the unit weight and the friction angle of the clay layer turned out to be the most influencing while also the cohesion of the Dikeold material and friction angle of the sand layer contribute to the failure domain. From a qualitative point of view, the weakening and consequently the settlement of the Dikeold material pushes the subsoil, creating an additional surcharge for the underlying clay and sand layer which act like the foundation soil; the incapability then of the clay layer to withstand the overlying load due to the low unit weight in combination with the low friction angle of the clay as well as the low stiffness and strength of the sand layer, can lead to the creation of an inner slip surface and thus failure. Since the clay layer can be considered as a foundation soil, the importance of the friction angle and the unit weight can be also explained from the Terzaghi's bearing capacity theory [16], where these two factors are crucial for the determination of the ultimate bearing capacity of the soil. However, these can be better verified by conducting large scale experiments in dikes and inspecting the sensitivity of the dike's stability towards the soil properties.

\subsection{System failure}

The general LSF for the system's reliability analysis is given in Eq. 10. In Table 8, the reliability analysis results are presented as they were deduced from DS. Similarly to the soil failure, in Table 9, the importance factors according to the Shortest Distance, the Average $10 \%$ and the Average all methods are illustrated.

\begin{tabular}{lc}
\hline \hline DS $\boldsymbol{P}_{\boldsymbol{f}}$ & $2.4 * 10^{-3}$ \\
$\beta$ & 3.0 \\
Number of LSF calls & 1807 \\
Number of iterations (or & 300 \\
directions) & 39 \\
Elapsed time (hr) & \\
\hline \hline
\end{tabular}

Table 8 Reliability results of DS for the system failure

The analysis took almost one and a half days and it came with indicative, valuable though, results about the system behaviour. According to the averaged importance factors in Table 9 (deduced by the Average all method), we can notice features from both soil and structural (anchor and sheet pile wall) failure. As far as the 
structural failure is concerned, the deduced importance factors from the anchor reliability analysis are given in Table 5. For the results of the sheet pile's reliability analysis, due to space limits, a reference is made to [14].

\begin{tabular}{l|ccc}
\hline \hline & $\beta_{\min }$ & Average 10\% & Average all \\
\hline \hline$G_{\text {clay }}$ & 0.014 & 0.148 & 0.183 \\
$c_{\text {clay }}$ & 0.040 & 0.037 & 0.021 \\
$\varphi_{\text {clay }}$ & 0.087 & 0.062 & 0.060 \\
Rinter $_{\text {clay }}$ & 0.052 & 0.035 & 0.070 \\
$\gamma_{\text {clay }}$ & 0.143 & 0.101 & 0.132 \\
$\varphi_{\text {sand }}$ & 0.029 & 0.025 & 0.075 \\
$G_{\text {sand }}$ & 0.047 & 0.051 & 0.062 \\
$c_{\text {dikeold }}$ & 0.000 & 0.039 & 0.081 \\
$\varphi_{\text {dikeold }}$ & 0.102 & 0.089 & 0.039 \\
$G_{\text {dikeold }}$ & 0.032 & 0.048 & 0.105 \\
Rinter $_{\text {dikeold }}$ & 0.407 & 0.281 & 0.040 \\
$\gamma_{\text {dikeold }}$ & 0.003 & 0.053 & 0.071 \\
\hline \hline
\end{tabular}

Table 9 Estimated importance factors $\alpha^{2}$ for the system failure with the different methods

For example, the unit weight (14\%), the friction angle $(7 \%)$ and the cohesion $(8 \%)$ of the clay layer as well as the friction angle $(8 \%)$ and the stiffness $(7 \%)$ of the sand layer that played an essential role in the soil failure analysis. Moreover, the stiffness of the clay (11\%) and the Dikeold (9\%) layers that appeared to be important for the anchor and sheet pile LSF. They seem to be also essential in the system analysis.

However, the other two methods (Shortest Distance and Average 10\%) came up with the cohesion and the interface strength of the Dikeold material to be the most influencing soil properties for the system. For that reason, an investigation was conducted regarding the output results of the system analysis in order to obtain an insight into the different reasons of the system failure. More precisely, the failure points were divided into those that come from Plaxis Error and those that come from the yield stress exceedance of the structural elements. It was then observed that many failure points originate from Plaxis errors that are related to "Not enough load steps" rather than "Soil seems to collapse".

Therefore, in order to estimate the actual important soil properties, the vectors of the random variables that led to errors of "Soil seems to collapse" and to anchor/sheet pile (SP) failure were studied separately. The importance factors are depicted in Table 10 which actually verifies the importance of the variables that were also deduced from the Averaged all method in Table 9 (which are basically the unit weight and the stiffness of the clay layer and the friction angle of the sand layer). It should be mentioned that the failure of the structural elements contributes $65 \%$ whereas the soil failure contributes $35 \%$, on the total probability of failure. The latter would be possibly lower than $2.4 * 10^{-3}$ that was found in Table 8 , as several failure points are not attributed to real failure but to Plaxis numerical errors.

It should be mentioned at that point, that in case of the anchor safety analysis that was presented in section 6.1 , such numerical errors were handled by making use of response surfaces. The main idea is that the response consisting of a complex function of input variables is approximated by a simple function of the input variables. In case of the anchor, this function was determined according to a sensitivity analysis. However, no further elaboration on that topic is made, as it is out of the scope of this paper. For more information, a reference is made to [14].

\begin{tabular}{l|cc}
\hline \hline & Anchor/SP & Soil collapse \\
\hline \hline$G_{\text {clay }}$ & 0.533 & 0.063 \\
$c_{\text {clay }}$ & 0.005 & 0.021 \\
$\varphi_{\text {clay }}$ & 0.094 & 0.098 \\
Rinter $_{\text {clay }}$ & 0.025 & 0.054 \\
$\gamma_{\text {clay }}$ & 0.114 & 0.188 \\
$\varphi_{\text {sand }}$ & 0.049 & 0.147 \\
$G_{\text {sand }}$ & 0.012 & 0.075 \\
$c_{\text {dikeold }}$ & 0.001 & 0.030 \\
$\varphi_{\text {dikeold }}$ & 0.028 & 0.039 \\
$G_{\text {dikeold }}$ & 0.062 & 0.111 \\
Rinter $_{\text {dikeold }}$ & 0.001 & 0.073 \\
$\gamma_{\text {dikeold }}$ & 0.028 & 0.041 \\
\hline \hline
\end{tabular}

Table 10 Estimated importance factors $\alpha 2$ for soil and the structural elements failure after the system analysis

The system analysis can lead to valuable conclusions for the system behaviour as a whole and individually for the different elements, given though that an inspection of the results is followed. Moreover, such an analysis is taking into account correlations among the three subfailure mechanisms implicitly. For example, the different LSFs may be affected by the same soil variable, such as the shear stiffness of the clay layer for the anchor and the sheet pile LSF. In that case, the most dominant failure mechanism will occur first and the rest are excluded.

\section{Conclusions and recommendations}

This paper presented a reliability analysis of a dike with an anchored sheet pile wall by making use of a soilstructure model in FEM. The output of the FEM calculations was utilized as an input in the probabilistic model. Different failure modes were analysed and the correspondent probability of failure and the influence factors were concluded.

As far as the reliability of the structural elements is concerned, their limit state was evaluated in terms of the exceedance of their yielding stress. The reliability assessment was carried out with FORM from which the probability of failure and the influence factors were concluded. More precisely, the anchor failure mode seemed to be more determinant than the soil failure, with the shear stiffness of the soft soil layers to be the most important soil properties. As it was proved by Rippi [14], the sheet pile failure is quite rare and it is unlikely to occur before the soil failure since the soil body has already entered its plastic domain. Therefore, anchor failure seems to be predominant.

The soil and the system failure were challenging to assess as there are multiple criteria that someone can choose. Regarding the soil failure, the Plaxis definition of soil collapse was used and it was evaluated with DS. In 
that case, the unit weight and the strength parameters of the soft soil layers seemed to be influencing. The detected failures were investigated in terms of their validity. That procedure revealed the possibilities and limitations of FEM modelling. The advantage is that a potential failure can be detected and its visualization is possible.

The failure of the system was considered as a serial system of the different LSFs and it was also assessed with DS. The analysis for system reliability showed that the importance factors stemmed from the reliability analysis indicated soil features that were important in all the elements. It should be stressed though that the failure probability of the system should be also coupled with the uncertainty of the water level and additional failure mechanisms (piping, internal erosion, etc.) should be investigated in order to obtain an overall picture of the total probability of failure. Further investigation is recommended for obtaining a more precise probability of failure after eliminating numerical errors in FEM calculations.

Nevertheless, this research proves the challenges and the potentials in a probabilistic reliability analysis. Such an analysis provides results required for design and assessment purposes, such as the probability of failure or the reliability index. A typical by-product is the so-called design point and the associated influence coefficients. The latter essentially indicates the contribution of each (random) variable to the total uncertainty. This information is useful to designers because they can infer from it if their focus should be on improving the structural design or reduce uncertainties in subsoil conditions. The influence coefficients can also give clear indications of which subsoil strata are dominant in the failure mechanisms at hand. Moreover, it is well-known that design recipes in codes of practices, including the corresponding partial factors, are conservative in terms of the actual reliability they produce on average, because they need to cover a wide range of structures and subsoil conditions. As a consequence, a fully probabilistic design should (in most cases) lead to a more economic design than the semi-probabilistic one.

\section{References}

1. Larsen H., Lubking P. and Breedeveld J. (2013). Ontwerp stabiliteitsschermen (type II) in primaire waterkeringen (groene versie). Referentie nummer 1205887-000-GEO-0016, Deltares. Delft, the Netherlands.

2. Orr TLL. (2000). Selection of characteristic values and partial factors in geotechnical designs to Eurocode 7. Computers and Geotechnics, 26, 263279.

3. Driscoll R. and Simpson B. (2001). EN1997 Eurocode 7: geotechnical design. Proceedings of the ICE-Civil Engineering, Vol. 144, No. 6, pp. 49-54

4. Delta programme. (2014). Synthesedocument veiligheid: Achtergronddocument B1. Ministrie van Infrastructuur en MilieuGouda, the Netherlands.

5. Schweckendiek T. (2006). Structural Reliability applied to Deep Excavations: Coupling Reliability
Methods with Finite Elements. MSc thesis, Delft University of Technology, Delft, Netherlands

6. Waarts P.H. (2000). Structural reliability using Finite Element Analysis: An appraisal of DARS (Directional Adaptive Response surface Sampling). Delft University of Technology, Delft, the Netherlands.

7. Möllman A. and Vermeer P. (2008). Reliability analysis of a dike failure: case study Elbe river. University of Stuttgart, Germany.

8. Bach D. (2014). Reliability-Based Design and Quality Control of Bored Piles. PhD thesis, Delft University of Technology, Delft, the Netherlands.

9. Hasofer A.M. and Lind N.C. (1974). An exact and invariant first-order reliability format. Journal of Engineering Mechanics Division, ASCE, 100(1), 111-121.

10. Vrijling J.K. and Gelder P.H.A.J.M. van. (2002). Probabilistic Design in Hydraulic Engineering, Lecture notes. Delft University of Technology, Faculty of Civil Engineering and Geosciences, Delft.

11. Baecher G.B. and Christian J.T. (2003). Reliability and Statistics in Geotechnical Engineering. Chichester, West Sussex: John Wiley \& Sons Ltd.

12. Griffiths D.V. and Fenton G.A (Eds.). (2007). Probabilistic Methods in Geotechnical Engineering. Lecture notes, monographs, edited works and proceedings in the field of Mechanics, Engineering, Computer Science and Applied Mathematics. Italy.

13. Phoon K. (Ed.). (2008). Reliability-based Design in Geotechnical Engineering: Computations and Applications. Taylor \& Francis, Britain.

14. Rippi A. (2015). Structural Reliability Analysis of a dike with a sheet pile wall: Coupling Reliability methods with Finite Elements. MSc Thesis. Department of Hydraulic Engineering, Delft University of Technology, the Netherlands.

15. Plaxis 2D. (2015). Reference Manual. The Netherlands.

16. Terzaghi K. (1942). Theoretical Soil Mechanics. New York: Wiley, ISBN 978-0-471-85305-3.

17. OpenTURNS 1.5. (2015). Reference Guide: OpenTURNS' methods for Step C: uncertainty propagation, pp.155-161.

18. Schweckendiek T. (2014). On Reducing Piping Uncertainties: A Bayesian Decision Approach. PhD thesis, University of Technology, Delft, Netherlands 